Revista Chilena
DE Pediatría

www.revistachilenadepediatria.cl

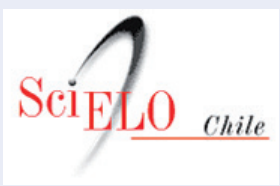

www.scielo.cl

\title{
Algunas consideraciones en la atención de niños y adolescentes trans
}

\author{
Some considerations in the management of children and \\ adolescents with non-conforming gender
}

\author{
Alejandro Martínez-Aguayo ${ }^{a}$, Mónica Arancibia Cabaláb, \\ Carolina Mendoza Fuentes ${ }^{\mathrm{c}}$
}

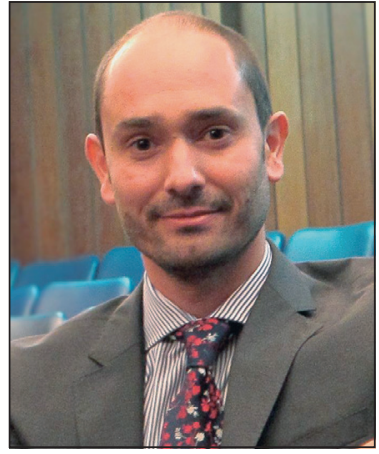

aDivisión de Pediatría, Pontificia Universidad Católica de Chile, Santiago de Chile

bServicio de Pediatría, Hospital Las Higueras, Talcahuano, Chile

cServicio de Pediatría, Hospital Clínico La Florida, La Florida, Chile

Un adolescente está en la sala de espera. La persona que lo atiende está confundida porque en la ficha clínica aparece un nombre de mujer que no concuerda con el "aspecto" del adolescente. El adolescente explica que su nombre social es diferente a su nombre legal. La persona que lo atiende insiste en usar el nombre legal durante el control de salud.

Transgénero es un término amplio para definir aquellos individuos, cuya identidad de género difiere del sexo asignado al nacer ${ }^{1,2}$. Si se considera como un espectro no binario (continuo o "paragua") es posible estimar una frecuencia de un 0,03 a 0,05\% de la población ${ }^{3}$. Algunas personas trans eligen realizar una transición social (ej. cambiar su nombre, pronombres, expresión de género) y/o transición médica (ej. tratamiento hormonal cruzado y/o cirugías), cuyo objetivo es alinear su expresión con su identidad de género, sin embargo no necesariamente los cambios elegidos van a estar dentro del tradicional género binario (masculino o femenino).

Las personas transgénero pueden tener que recorrer un largo camino para acceder a una atención de salud adecuada en relación a su condición de sujetos trans. Por este motivo evitan con frecuencia la atención médica, convirtiéndose en una población altamente vulnerable, por lo que es importante recodar algunos conceptos y aspectos éticos relacionados con el acompañamiento de estas personas.

Primero que nada es importante que los profesionales de la salud comprendan las diferencias entre sexo y género y que todos los pacientes merecen ser tratados con el máximo de respeto, sensibilidad y consideración. De acuerdo a los niños y adolescentes con género no conforme, es deseable que el equipo médico entienda al menos estos puntos: "Sexualidad/orientación sexual y género cosas son totalmente diferentes", "Hablar algunas cosas con extraños es difícil", "Existen personas no binarias", "Nombre y pronombres son importantes", "Es mejor preguntar que asumir", "No pregunte por los genitales, a menos que sea medicamente necesario", "El examen de genitales puede ser muy incómodo", "Bloqueadores de pubertad / tratamiento hormonal cruzado pueden salvar mi vida", "Usen mi nombre social, y si comete un error, no se sobre-disculpe", "No estoy deprimido por ser trans, la sociedad me deprime y estigmatiza" y "Hazme saber que estás de mi lado"4.

Correspondencia:

Alejandro Martínez-Aguayo

alemarti@med.puc.cl 
Las personas involucradas en la atención de niños y adolescentes con género no conforme también son susceptibles a la estigmatización, que es un proceso social complejo y dinámico, en el cual se etiqueta, estereotipa y se rechazan las diferencias humanas como una forma de control social ${ }^{3}$. La estigmatización puede ocurrir en tres niveles: a) "Estructural", como políticas institucionales y/o normas sociales que afecten el acceso a la salud de las personas (por ejemplo: que un hombre-trans no pueda tener control de embarazo o poder realizar tamizaje de cáncer de cuello uterino); b) "Interpersonal", como violencia verbal y/o física (por ejemplo, rechazo a tratar al sujeto trans por el nombre social y pronombres con los que se sienta identificado nuestro paciente, a hacer responsable a la persona por alguna patología que ésta padezca), y c) "Individual", este es el estigma que la propia persona tiene como vivencia o los sentimientos que provocan otras personas en él. El estigma puede perjudicar la salud de una persona trans generando estrés y haciendo más difícil el acceso a los sistemas de salud, aumentando el riesgo de depresión, ansiedad, riesgo de auto-agresión y suicidio ${ }^{5}$.

Basado en los principios éticos de autonomía, nomaleficencia, beneficencia y justicia, Hann y colaboradores ${ }^{6}$ han sugerido eliminar algunas de las barreras a las que las personas trans están expuestas. Autonomía: Las personas trans tienen derecho a que su identidad sea respetada por el equipo médico, el nombre social e identidad de género deben ser registradas en la historia clínica. Deben participar en las decisiones de salud bajo el régimen de "consentimiento informado", las decisiones de iniciar tratamientos hormonales / quirúrgicos deben ser respetadas y registradas confidencialmente en la ficha clínica. No-maleficencia: Evitar crear barreras en la atención médica, no estigmatizar y no discriminar, a veces es difícil solicitar algunos exámenes de imágenes o bioquímicos cuando los procedimientos han sido asignados exclusivamente en base a género binario (por ejemplo, tamizaje de cáncer de próstata-hombres y de cuello uterino-mujeres). Los valores de referencia para los exámenes bioquímicos no consideran a las personas trans que están bajo tratamiento hormonal cruzado, la persona que interprete los resultados debe hacerlo con cautela (por ejemplo, hemograma o densitometría ósea). Beneficencia: El equipo de salud debe tener una aproximación "afirmativa”, de compresión y desprejuiciada de la persona. Las personas que reciben una aproximación afirmativa, mejoran en aproximadamente un $80 \%$ su calidad de vida e incluso disminuyen algunas condiciones de salud mental ${ }^{5}$. Justicia: Las personas trans tienen derecho a una distribución justa de los recursos sanitarios. En algunos países podrían tener menor cobertura en salud que las personas cis (personas que tienen identidad de género igual a la asignada al nacer) y las terapias con hormonas cruzadas, cirugías y los seguimientos necesarios por un equipo interdisciplinario pueden no estar financiados por el estado o compañías aseguradoras.

En enero del año 2018, el Hospital Sótero del Río inauguró el primer "Programa de Identidad de Género" en la Región Metropolitana. Esta inauguración para formalizar la atención a adultos transgénero, que ya se realizaba en ese centro hace algunos años, contó con el apoyo del SSMSO, lo que permitió obtener recursos específicos para el funcionamiento del programa. Sin embargo, no contemplaba la atención de menores de 15 años, ya que este equipo no contaba con un endocrinólogo pediatra. Durante la inauguración del programa, se invitó a participar a un pediatra endocrinólogo de manera de complementar la atención de endocrinología de adultos con la de pediatría. Desde esa fecha, se ha trabajado de manera conjunta en un grupo interdisciplinario, generando derivaciones internas entre los distintos profesionales. Hasta junio del 2019 se atienden aproximadamente 370 usuarios, de los cuales 90 son menores de 18 años y 12 menores de 15 años. Otro ejemplo es el del Hospital Las Higueras de Talcahuano, el que desde el año 2012 atiende a personas trans adultos. El 2014 se creó formalmente el "Programa de Atención a Usuarios Trans del Hospital Las Higueras" que permanece activo hasta el día de hoy. Dada la necesidad de atención a niños y adolescentes trans, en diciembre de 2018 se diseñó un plan de trabajo interdisciplinario (en el mismo Hospital de la Higueras) para crear un protocolo de atención que fue formalizado en marzo de 2019. La atención se inició en abril del mismo año, y hasta junio de 2019, hay 88 usuarios en control, 5 de ellos menores de 15 años.

En mayo 2019, se realizó en el Hospital Las Higueras la "Primera Jornada de Capacitación en Atención de Pacientes Trans Pediátricos" con el objetivo de educar sobre el abordaje integral del niño y adolescente trans e informar sobre la existencia del programa de atención a usuarios trans del Hospital. A esta actividad, organizada por pediatras endocrinólogos del Hospital Las Higueras asistieron más de 150 profesionales de la salud, principalmente funcionarios de la Red de Salud de Talcahuano.

El equipo de salud mental debe ser experto en terapia trans-afirmativa, respetuoso y que apoyen los procesos de experiencia de vida de las personas trans. Además es necesario tener una aproximación basada en aspectos culturales y sociales, con el objetivo de desarrollar una buena relación entre los proveedores de salud y el usuario, mejorando la experiencia de ambos. Es necesario que existan centros altamente especializados para la evaluación, manejo y acompañamiento interdisciplinario de las personas trans como son los ejemplos aquí referidos. 
Tomando en cuenta la complejidad relacionada a los temas de identidad de género y en especial de sujetos trans, es necesario constituir equipos de atención médica que se familiaricen y se hagan expertos en terapia trans-afirmativa, de manera de apoyar con respeto los procesos vitales de las personas trans. Es imprescindible contemplar en la aproximación del tratamiento, aspectos culturales y sociales, con el objetivo de desarrollar una buena relación entre los proveedores de salud y el usuario, mejorando la experiencia de ambos. Además, es necesario crear más centros altamente especializados para la evaluación, manejo y acompañamiento interdisciplinario de las personas trans, como los ejemplos aquí referidos.

\section{Conflicto de intereses}

Los autores declaran no tener conflicto de intereses.

\section{Referencias}

1. Davidson M. Seeking refuge under the umbrella: Inclusion, exclusion, and organizing within the category Transgender. Sex Res Soc Policy. 2007;

2. Jauk D, Feltey K. Imagining Transgender: An Ethnography of a Category. Sex Roles. 2010;

3. White Hughto JM, Reisner SL, Pachankis JE. Transgender stigma and health: A critical review of stigma determinants, mechanisms, and interventions. Social
Science and Medicine. 2015.

4. Turban J, Ferraiolo T, Martin A, Olezeski C. Ten Things Transgender and Gender Nonconforming Youth Want Their Doctors to Know. Journal of the American Academy of Child and Adolescent Psychiatry. 2017.

5. Hembree WC, Cohen-kettenis PT, Gooren L, et al. Endocrine Treatment of Gender-Dysphoric/Gender-Incongruent Persons: An Endocrine Society Clinical Practice GuidelineCL IN IC A L Endocrine Treatment of Gender-
Dysphoric / Gender-Incongruent Persons: An Endocrine Society * Clinical Practice Guideline. J Clin Endocrinol Metab. 2017;102(February):3869-903.

6. Hann M, Ivester R, Denton GD Bioethics in Practice: Ethical Issues in the Care of Transgender Patients. Ochsner J [Internet]. 2017;17(2):144-5. Available from: http://www.ncbi.nlm.nih. gov/pubmed/28638286\%0Ahttp://www. pubmedcentral.nih.gov/articlerender. fcgi?artid=PMC5472072. 